\title{
Composição química e avaliação das atividades antibacteriana e de toxicidade dos óleos essenciais de Lantana camara $\mathrm{L}$. e Lantana sp.
}

\author{
José G. M. da Costa, ${ }^{* 1}$ Erlânio O. de Sousa, ${ }^{1}$ Fabíola F. G. Rodrigues, ${ }^{1}$ \\ Sidney G. de Lima, ${ }^{2}$ Raimundo Braz-Filho ${ }^{3}$ \\ ${ }^{1}$ Programa de Pós-graduação em Bioprospecção Molecular, Laboratório de Pesquisas de Produtos Naturais, \\ Universidade Regional do Cariri, Bairro Pimenta, 63105-000 Crato-CE, Brasil \\ ${ }^{2}$ Departamento de Química, Universidade Federal do Piauí, Campus Universitário Ministro Petrônio Portella, \\ Bairro Ininga, 64049-550 Teresina-PI, Brasil \\ ${ }^{3}$ Programa de Pós-Graduação em Ciências Naturais, Universidade Estadual do Norte Fluminense Darcy Ribeiro, \\ Campus dos Goytacazes, 28013-602 Rio de Janeiro-RJ, Brasil
}

\begin{abstract}
RESUMO: Os óleos essenciais de Lantana camara L. e Lantana sp., Verbenaceae, foram obtidos por hidrodestilação e seus componentes analisados por CG/EM. Tal procedimento analítico revelou a predominância dos sesquiterpenos, biciclogermacreno $(19,42 \%)$, isocariofileno $(16,70 \%)$, valenceno (12,94\%) e germacreno D (12,34\%) no óleo de L. camara, enquanto o biciclogermacreno (13,93\%), germancreno D (27,54 e $\beta$-cariofileno (31,50\%), \%) destacaram-se como majoritários no óleo de Lantana sp. Os dois óleos foram submetidos a avaliação de atividades antibacteriana e de toxicidade usando as larvas de Artemia salina. Os resultados mostraram considerável atividade antibacteriana do óleo de L. camara, principalmente para Proteus vulgaris (ATCC 13315) e Escherichia coli (ATCC 25922). O óleo de Lantana sp. mostrou-se mais significativo contra Sthaphylococcus aureus (ATCC 10390). As atividades de toxicidade $\mathrm{CL}_{50}$ revelaram resultados inferiores ao limite padrão de $1000 \mu \mathrm{g} / \mathrm{mL}$.
\end{abstract}

Unitermos: Lantana, Verbenaceae, óleo essencial, terpernóides, atividades biológicas.

\begin{abstract}
Chemical composition, evaluation of antibacterial activity and toxicity of the essential oils from Lantana camara L. and Lantana sp.". The essential oils from Lantana camara L. and Lantana sp. Verbenaceae, were obtained by hydrodistillation and analyzed by GC/MS. This GC/MS analysis revealed the sesquiterpenoids bicyclogermacrene (19.42\%), isocaryophyllene (16.70\%), valencene (12.94\%) and germacrene D (12.34\%) as majoritaries in the essential oil of $L$. camara, and bicyclogermacrene (13.93\%), germacrene D (27.54 and $\beta$-caryophyllene $(31.50 \%)$ in the essential oil from Lantana sp. The two essential oils were evaluated to antibacterial activity and toxicity using Artemia salina. The results revealed considerable antibacterial activity for essential oil from L. camara, meanly for Proteus vulgaris (ATCC 13315) and Escherichia coli (ATCC 25922). The essential oil of Lantana sp. showed major activity for Sthaphylococcus aureus (ATCC 10390). The evaluation of toxicity $\mathrm{CL}_{50}$ revealed results with minor values to limit pattern of 1000 $\mu \mathrm{g} / \mathrm{mL}$.
\end{abstract}

Keywords: Lantana, Verbenaceae, essential oils, terpernoids, biological activities.

\section{INTRODUÇÃO}

O gênero Lantana apresenta cerca 150 espécies descritas, sendo a maioria cultivada com objetivo decorativo devido à beleza de suas flores. Entretanto são geralmente pouco palatáveis e nem todas são tóxicas (Joly, 1993). Estas plantas são praticamente imunes ao ataque de herbívoros, devido à presença de uma grande diversidade de substâncias naturais (Kohli et al., 2006).

Lantana camara L., Verbenaceae, é uma planta que ocorre em muitas regiões tropicais e subtropicais do mundo. É usada na medicina popular como emenagoga, diurética, expectorante, febrífuga e anti-reumática (Agra et al., 2007; 2008; Brandão et al., 2009; Leitão et al., 2009). Suas raízes e folhas são atribuídas propriedades anticonvulsivante (Quintans-Júnior et al., 2008). As folhas são ricas em óleos essenciais, compostos fenólicos e triterpenóides, com predominância de flavonóides. Tais substâncias presentes nessa espécie também são encontradas em algumas plantas daninhas que possui 
propriedades alelopáticas e de toxicidade (Sharma et al., 2007).

A ingestão de folhas de Lantana por animais causa colestase e hepatotoxicidade. Animais ruminantes e não-ruminantes como cobaias, coelhos e fêmeas de ratos apresentaram suscetibilidade à ação de hepatotoxinas, que são triterpenóides pentacíclicos denominados de lantadenos (Kohli et al., 2006). Os frutos da planta também são tóxicos para o homem.

Neste trabalho estão sendo relatadas as composições, as investigações de atividades antibacterianas e de toxicidade, e a comparação dos óleos essenciais de duas espécies de Lantana provenientes do Cariri Cearense. A análise dos dois óleos essenciais por cromatografia gasosa (CG) acoplada a espectrômetro de massas (EM) permitiu identificar os constituintes majoritários no óleo de Lantana sp.

\section{MATERIAL E MÉTODOS}

\section{Material vegetal}

As folhas das espécies Lantana camara L. e Lantana sp. foram coletadas em junho de 2007, no Horto de Plantas Aromáticas e Medicinais do Laboratório de Pesquisas de Produtos Naturais na Universidade Regional do Cariri, Crato-CE. Exsicatas das duas espécies foram depositadas no Herbário Caririensis Dárdaro de Andrade Lima do Departamento de Biologia desta IES, com registros 1662 e 1619, respectivamente.

\section{Extração dos óleos essenciais}

Os óleos essenciais foram obtidos separadamente, partindo-se de $200 \mathrm{~g}$ de folhas frescas trituradas e utilizando-se o sistema de hidrodestilação, em aparelho tipo Clevenger (Matos et al., 1999) durante o período de duas horas. Os óleos coletados foram subseqüentemente secos com sulfato de sódio anidro $\left(\mathrm{Na}_{2} \mathrm{SO}_{4}\right)$ e mantidos sob refrigeração até a realização das análises. Os rendimentos dos óleos foram 0,05\% para Lantana camara e $0.07 \%$ para Lantana sp., calculados com base nos volumes de óleos obtidos e dos pesos dos materiais vegetais frescos.

\section{Análise por CG/EM}

As análises dos constituintes voláteis foram realizadas em equipamento CG/EM Hewlett-Packard, Modelo 5971 usando-se coluna capilar não-polar DB-1, de sílica fundida (30 m x 0,25 mm i.d., película de $0,25 \mu \mathrm{m})$; carreado por gás hélio; velocidade de fluxo $0,8 \mathrm{~mL} / \mathrm{min}$ e modo de divisão. A temperatura do injector e do detector foram $250{ }^{\circ} \mathrm{C}$ e $200{ }^{\circ} \mathrm{C}$, respectivamente. A temperatura da coluna foi programada de $35^{\circ} \mathrm{C}$ para $180{ }^{\circ} \mathrm{C} \mathrm{em} 4{ }^{\circ} \mathrm{C} /$ min e em seguida $180{ }^{\circ} \mathrm{C}$ para $250{ }^{\circ} \mathrm{C}$ em $10^{\circ} \mathrm{C} / \mathrm{min}$. Os espectros de massas foram gravados a partir de 30-450 $\mathrm{m} / \mathrm{z}$. Componentes individuais foram identificados por correspondência de seus espectros de massa, $70 \mathrm{eV}$, com os da base de dados usando a biblioteca construída através do espectrômetro Wiley e outros dois computadores utilizando índices de retenção como uma pré-seleção (Alencar et al., 1984; Alencar et al., 1990), bem como por comparação visual da fragmentação padrão com aqueles relatados na literatura (Stenhagen et al., 1974; Adams, 2001).

\section{Ensaio antibacteriano}

Os ensaios antibacterianos foram realizados pelo método de difusão em disco (Bauer et al., 1966). Para tanto, foram utilizadas cinco culturas de bactérias, cedidas pela Fundação Oswaldo Cruz, sendo quatro gram-negativas (Escherichia coli (ATCC 25922), Proteus vulgaris (ATCC 13315), Pseudomonas aeruginosa (ATCC 15442) e Vibriu cholareal (ATCC 15748)) e uma gram-positiva (Sthaphylococcus aureus (ATCC 10390)). Essas foram inoculadas em ágar Brain Heart Infusion e incubadas por $24 \mathrm{~h} / 37^{\circ} \mathrm{C}$ para reavivamento. Após esse período foram semeadas em placa com ágar Muller-Hilton, utilizando swabs estéreis. Discos de papel de filtro estéreis $(6 \mathrm{~mm}$ de diâmetro) impregnados com $20 \mu \mathrm{L}$ das soluções dos óleos $(10 ; 5 ; 2,5 ; 1,25 ; 0,62 \%)$. As placas foram incubadas em estufa a $37^{\circ} \mathrm{C}$ e por $24 \mathrm{~h}$ e após esse período examinadas quanto à presença de halos de inibição. Os ensaios foram realizados em triplicata e acompanhados de controles positivos com discos comerciais, cloranfenicol $(30 \mu \mathrm{g})$ e azitromicina $(15 \mu \mathrm{g})$ e controle negativo, DMSO e água destilada.

\section{Ensaio de toxicidade}

Nos ensaios de toxicidade utilizou-se a metodologia preconizada por Meyer e colaboradores (Meyer et al., 1982), frente à Artemia salina Leach. Os ensaios realizaram-se em triplicata com diferentes concentrações $(100 ; 50 ; 25 ; 10 \mu \mathrm{g} / \mathrm{mL})$, seguidos de controle positivo preparado com água marinha e dicromato de potássio $\left(\mathrm{K}_{2} \mathrm{Cr}_{2} \mathrm{O}_{7}\right)$, e controle negativo com água marinha e Tween 80. As contagens dos números de larvas mortas foram realizadas após $24 \mathrm{~h}$ e utilizou-se esse número para o calculo da $\mathrm{CL}_{50}$ pelo método de regressão linear, sendo considerado ativo quando $\mathrm{CL}_{50}<1000 \mu \mathrm{g} /$ $\mathrm{mL}$.

\section{RESULTADOS E DISCUSSÃO}

As análises dos óleos essenciais por CG/ EM possibilitaram reconhecer a presença de mono- e sesquiterpenos como componentes predominantes em ambas as espécies. No óleo de L. camara foi possível identificar 25 constituintes, representando 99,75\%, enquanto que no óleo de Lantana sp. identificou-se dezenove constituintes totalizando 98,65\% (Tabela 1). 
Os constituintes químicos majoritários no óleo de L. camara em ordem de abundância foram: biciclogermacreno $(\mathbf{1}, 19,42 \%)$, isocariofileno $(\mathbf{2}, 16,70$ $\%)$, valenceno $(3,12,94 \%)$ e germacreno D $(4,12,34$ \%). As substâncias identificadas como predominantes no óleo de Lantana sp. foram: $\beta$-cariofileno $(\mathbf{5}, 31,50 \%)$, germancreno $\mathrm{D}(4,27,54 \%)$ e biciclogermacreno $(1,13,93$ $\%)$.

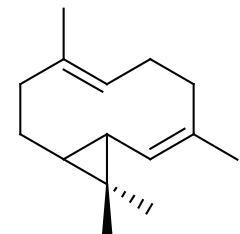

1

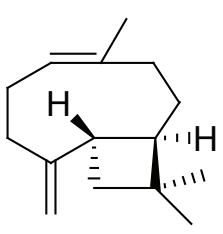

2<smiles>C=C(C)[C@@H]1CCC2=CCC[C@H](C)[C@]2(C)C1</smiles>

3<smiles>C=C(C)CC/C(C)=C\CC[C@@H](/C=C/C(=C)CC)C(C)C</smiles>

4

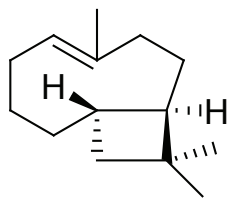

5
Os ensaios de atividade antimicrobiana in vitro mostraramoóleo de L. camara comoinibidordocrescimento de quase todas as bactérias testadas, aparecendo a $S$. aureus como resistente à ação dos componentes presentes no óleo. Os melhores resultados foram obtidos nos testes com a $P$. vulgaris e E. coli, observando-se halos de 9.5 $\mathrm{mm}$ e $9 \mathrm{~mm}$, respectivamente (Tabela 2). Nos ensaios com óleo de Lantana sp. verificou-se sensibilidade de todas as bactérias, ocorrendo destaque para $S$. aureus com halo de $12 \mathrm{~mm}$. As bactérias E. coli e P. aeruginosa, apresentaram resultados semelhantes com halos de $11 \mathrm{~mm}$ (Tabela 3). As análises estatísticas (Tabelas 2 e 3 ) foram realizadas no programa Microsoft Excel for Windows (Neufeld, 2003).
Segundo Ohno et al., 2003, existem diferenças na atividade bacteriana entre os óleos essenciais do mesmo gênero. Essa diferença está relacionada principalmente pela variabilidade de quimiotipos, ciclo vegetativo, fatores extrínsecos e processo de obtenção desses óleos. Conforme Koyama et al. (1997), muitos componentes dos óleos essenciais, os quais são diferentes em cada óleo, possuem
Tabela 1. Componentes identificados nos óleos essenciais das folhas de Lantana camara (óleo I) e Lantana sp. (óleo II), com as referidas porcentagens. * RT $=$ Tempo de Retenção

\begin{tabular}{|c|c|c|c|c|}
\hline \multirow{2}{*}{ Componentes } & \multicolumn{2}{|c|}{ Óleo I } & \multicolumn{2}{|c|}{ Óleo II } \\
\hline & $\mathrm{RT}^{*}$ & $(\%)$ & $\mathrm{RT}^{*}$ & $(\%)$ \\
\hline Z-Ocimeno & 9,49 & 0,94 & - & - \\
\hline Sabineno & 10,76 & 4,78 & 9,55 & 0,22 \\
\hline 2- $\beta$-Pineno & 10,89 & 0,25 & - & - \\
\hline$\beta$-Mirceno & 11,40 & 0,39 & - & - \\
\hline$E$ - $\beta$-Ocimeno & 12,17 & 1,02 & - & - \\
\hline$p$-Ment-2-en-1-ol & 12,75 & 5,17 & - & - \\
\hline$\beta$-Cimeno & 13,49 & 1,37 & - & - \\
\hline$\gamma$-Terpineno & 13,86 & 0,62 & - & - \\
\hline Linalol & - & - & 14,14 & 1,83 \\
\hline$\alpha$-Terpinoleno & 15,24 & 0,83 & & - \\
\hline Cânfora & - & - & 15,71 & 0,34 \\
\hline 4-Terpineol & 18,03 & 0,59 & - & - \\
\hline$\alpha$-Copaeno & 25,75 & 0,42 & 24,26 & 2,70 \\
\hline$\beta$-Elemeno & 26,19 & 1,16 & 24,76 & 2,93 \\
\hline$\beta$-Cariofileno & 29,41 & 2,91 & 25,78 & 31,50 \\
\hline$\alpha$-Humulene & 27,02 & 2,07 & 26,83 & 2,68 \\
\hline Iso-cariofileno & 27,22 & 16,70 & - & - \\
\hline
\end{tabular}

uma habilidade específica diferenciada para romper ou penetrar na estrutura bacteriana. Para se conhecer o modo de ação dos constituintes presentes nos óleos de L. camara e Lantana sp. será necessário examinar isoladamente cada constituinte, e a sincronização entre eles, para averiguar atividades bacterianas.

Os ensaios de toxicidade com $A$. salina mostraram que os óleos essenciais das duas espécies exibiram atividades similares e significativas, revelando $14 \mu \mathrm{g} /$ $\mathrm{mL}$ para L. camara e $24 \mu \mathrm{g} / \mathrm{mL}$ para Lantana sp. como as doses calculadas necessárias para matar $50 \%\left(\mathrm{CL}_{50}\right)$. Tais valores indicam fortes atividades de toxicidade por serem inferiores ao limite padrão de $1000 \mu \mathrm{g} / \mathrm{mL}$ (Tabela 4).

\begin{tabular}{|c|c|c|c|c|}
\hline Aloaromadandreno & 28,13 & 2,29 & 27,07 & 1,37 \\
\hline$\beta$-Sabineno & - & - & 27.90 & 0,73 \\
\hline Biciclogermacreno & 28,45 & 19,42 & 28,24 & 13,93 \\
\hline Germacreno A & - & - & 28,50 & 1,08 \\
\hline Torreiol & - & - & 28,78 & 1,33 \\
\hline$\beta$-Cadineno & - & - & 29,01 & 2,59 \\
\hline Germacreno D & 29,35 & 12,34 & 29,91 & 27,50 \\
\hline Valeceno & 29,90 & 12,94 & - & - \\
\hline$\gamma$-Cadineno & 30,06 & 0,49 & - & - \\
\hline Germacreno B & - & - & 30,12 & 1,43 \\
\hline$\alpha$-Elemeno & 31,32 & 1,59 & - & - \\
\hline Espatulenol & 31,83 & 2,58 & 30,69 & 3,37 \\
\hline$\gamma$-Elemeno & 31,41 & 1,59 & - & - \\
\hline$\alpha$-Cadinol & - & - & 32,56 & 0,51 \\
\hline Oxido de cariofileno & 32,95 & 1,73 & 30,87 & 2,21 \\
\hline$\Delta$-Cadinol & - & - & 32,96 & 0,40 \\
\hline Azulenol & 33,06 & 5,56 & - & - \\
\hline Total & & 99,75 & & 98,65 \\
\hline
\end{tabular}

*RT = Tempo de Retenção 
Tabela 2. Resultados dos halos de inibição para a atividade antibacteriana do óleo essencial de Lantana camara.

\begin{tabular}{|c|c|c|c|c|c|c|c|c|}
\hline \multirow{3}{*}{ Bactérias } & \multicolumn{8}{|c|}{ Média dos Halos de Inibição $(\mathrm{mm}) \pm \mathrm{DP}$} \\
\hline & \multicolumn{5}{|c|}{ Concentração do óleo I (\%) } & \multicolumn{3}{|c|}{ Controles } \\
\hline & 10 & 5 & 2,5 & 1,25 & 0,62 & CLO & AZI & $\mathrm{AeT}$ \\
\hline S. aureus & ND & ND & ND & ND & ND & $12,5 \pm 0,70$ & $11,33 \pm 1,52$ & ND \\
\hline E. coli & $9,0 \pm 0,00$ & $8,5 \pm 0,70$ & $7,5 \pm 0,70$ & $7,0 \pm 0,00$ & $6,5 \pm 0,70$ & ND & $14,66 \pm 1,52$ & ND \\
\hline P. vulgaris & $9,5 \pm 0,70$ & $9,0 \pm 0,00$ & $7,5 \pm 0,70$ & $6,5 \pm 0,70$ & $6,5 \pm 0,70$ & $16,0 \pm 1,73$ & $15,33 \pm 1,00$ & ND \\
\hline P. aeruginosa & $75,5 \pm 0,70$ & $7,0 \pm 1,41$ & $7,0 \pm 0,00$ & $6,5 \pm 0,70$ & $6,0 \pm 0,00$ & ND & $14,66 \pm 0,00$ & ND \\
\hline V. cholareal & $8,5 \pm 0,70$ & $7,5 \pm 0,70$ & $7,0 \pm 0,00$ & $6,5 \pm 0,70$ & $6,0 \pm 0,00$ & ND & ND & ND \\
\hline
\end{tabular}

$\mathrm{ND}=$ Não detectável; $\mathrm{CLO}=$ cloranfenicol $(30 \mu \mathrm{g} / \mathrm{mL}) ; \mathrm{AZI}=$ azitromicina $(15 \mu \mathrm{g} / \mathrm{mL}), \mathrm{A}$ e T $=$ água e Tween.

Tabela 3. Resultados dos halos de inibição para a atividade antibacteriana do óleo essencial de Lantana sp.

\begin{tabular}{|c|c|c|c|c|c|c|c|c|}
\hline \multirow{3}{*}{ Bactérias } & \multicolumn{8}{|c|}{ Média dos Halos de Inibição $(\mathrm{mm}) \pm \mathrm{DP}$} \\
\hline & \multicolumn{5}{|c|}{ Concentração do óleo II (\%) } & \multicolumn{3}{|c|}{ Controles } \\
\hline & 10 & 5 & 2,5 & 1,25 & 0,62 & CLO & AZI & $\mathrm{Ae} \mathrm{T}$ \\
\hline S. aureus & $11 \pm 0,70$ & $9 \pm 0,00$ & $7 \pm 0,70$ & $7 \pm 0,00$ & $6 \pm 0,00$ & ND & $14,66 \pm 1,52$ & ND \\
\hline E. coli & $11,5 \pm 0,70$ & $9,5 \pm 0,70$ & $9 \pm 0,00$ & $8,5 \pm 0,70$ & $7,5 \pm 0,70$ & $16 \pm 1,73$ & $15,33 \pm 1,00$ & ND \\
\hline P. vulgaris & $11 \pm 0,00$ & $9.5 \pm 0,70$ & $9 \pm 0,00$ & $7,5 \pm 0,70$ & $7 \pm 0,00$ & ND & $14,66 \pm 0,00$ & ND \\
\hline P. aeruginosa & $9 \pm 1,41$ & $9 \pm 0,00$ & $8 \pm 1,41$ & $7,5 \pm 0,70$ & $6 \pm 0,00$ & ND & ND & ND \\
\hline V. cholareal & $12 \pm 0,00$ & $11 \pm 0,00$ & $9,5 \pm 0,70$ & $9,5 \pm 0,70$ & $8 \pm 1,41$ & $12,5 \pm 0,70$ & $11,33 \pm 1,52$ & ND \\
\hline
\end{tabular}

$\mathrm{ND}=$ Não detectável; $\mathrm{CLO}=$ cloranfenicol $(30 \mu \mathrm{g} / \mathrm{mL}) ; \mathrm{AZI}=$ azitromicina $(15 \mu \mathrm{g} / \mathrm{mL}), \mathrm{A}$ e T $=$ água e Tween.

Tabela 4: Percentual de mortalidade das larvas de Artemia salina, frente os óleos essencial das folhas de Lantana camara (óleo I) e Lantana sp. (óleo II).

\begin{tabular}{ccc}
\multirow{2}{*}{ Concentração $(\mu \mathrm{g} / \mathrm{mL})$} & \multicolumn{2}{c}{ Mortalidade das larvas $(\%)$} \\
\cline { 2 - 3 } & Óleo I & Óleo II \\
\hline 100 & 99,0 & 99,9 \\
50 & 86,7 & 69.0 \\
25 & 56,7 & 43,3 \\
10 & 36,7 & 26,0 \\
\hline
\end{tabular}

\section{AGRADECIMENTOS}

Ao CNPq, CAPES, FUNCAP e FAPERJ pelas bolsas e auxílios financeiros concedidos e a Fiocruz pela concessão das cepas bacterianas.

\section{REFERÊNCIAS}

Adams RP 2001. Identification of essencial oil components by gas chromatography/massa spectroscopy. Baylor University. Allured.

Agra MF, França PF, Barbosa-Filho JM 2007. Synopsis of the plants known as medicinal and poisonous in Northeast of Brazil. Rev Bras Farmacogn 17: 114-140.
Agra MF, Silva KN, Basílio IJLD, França PF, Barbosa-Filho JM 2008. Survey of medicinal plants used in the region Northeast of Brazil. Rev Bras Farmacogn 18: 472-508.

Alencar JW, Craveiro AA, Matos FJA 1984. Kovats' indices as a preselection routine in mass spectra library search of volatiles. J Nat Prod 47: 890-892.

Alencar JW, Craveiro AA, Matos FJA, Machado MIL 1990. Kovats indices simulation in essential oils analysis. Quim Nova 13: 282-284.

Bauer AW, Kirby WMM, Sherris JC, Turck M 1966. Antibiotic susceptibility festing by a standartized single disc melhod. Am J Clin Patol 45: 493-96.

Brandão MGL, Cosenza GP, Grael CFF, Monte-Mór RLM 2009. Traditional uses of American plant species from the 1st edition of Brazilian Official Pharmacopoeia. Rev Bras Farmacogn 19: 478-487.

Joly AB 1993. Introdução à Taxonomia Vegetal, $11^{\mathrm{a}}$ ed., Companhia Editora Nacional: São Paulo.

Kohli RK, Batish DR, Batish HP, Singh HP, Dogra KS 2006. Status, invasiveness and environmental threats of three tropical American invasive weeds (Parthenium hysterophorus L., Ageratum conyzoides L., Lantana camara L.) in India. Biol Invasions 8: 1501-1510.

Koyama S, Yamaguchi Y, Tanaka S, Motoyashima J 1997. A new substance (yoshixol) with na interesting antibiotic mechanism from wood oil of Japanase traditional tree (kisohinoki), Chamaecyparis obtusa. Gen Pharmacol 
28: 797-804.

Leitão F, Fonseca-Kruel VS, Silva IM, Reinert F 2009. Urban ethnobotany in Petrópolis and Nova Friburgo (Rio de Janeiro, Brazil). Rev Bras Farmacogn 19: 333-342.

Matos FJA, Machado MIL, Craveiro AA, Barbosa-Filho JM 1999. Essential oil of Mentha x villosa Huds. J Essent Oil Res 11: 41-44.

Meyer BN, Ferrigne NR, Putnam JE, Jacobsen LB, Nichols DE, Mclaughlin JE 1982. Brine Shrimp: A convenient general bioassay for active plant constituents. Planta Med 45:3134.

Neufeld JL2003. Estatística aplicada à administração usando Excel; tradução José Luiz Celeste; São Paulo: Prentice Hall.

Ohno T, Kita M, Yamaoka Y, Imamura S, Yamamoto SM, Kodama T, Kashima K, Imanishi J 2003. Antimicrobial activity of essential oils against Helicobacter pylori. Helicobacter 8: 207-215.

Quintans-Júnior LJ, Almeida JRGS, Lima JT, Nunes XP, Siqueira JS, Oliveira LEG, Almeida RN, Athayde-Filho PF, Barbosa-Filho JM 2008. Plants with anticonvulsant properties - a review. Rev Bras Farmacogn 18 (Supl.): 798-819.

Sharma POM, Sarita S, Vasantha P, Shashi BM, Pritam DS 2007. A Review of the hepatotoxic plant Lantana camara. Clin Toxicol 37: 313-352.

Stenhagen E, Abrahamson S, McLafferty FW 1974. Registry of Mass Spectra Data Base, Washington DC: Government Printing Office. 